\title{
Agôn
}

Revue des arts de la scène

Critiques | Saison 2011-2012

\section{Pouvoir et Corps : quel corps?}

Jusque là c'est nous, Matthieu Hocquemiller

\section{Barbara Métais-Chastanier}

\section{(2) OpenEdition}

Journals

Édition électronique

URL : http://journals.openedition.org/agon/2275

DOI : 10.4000 /agon.2275

ISSN : 1961-8581

Éditeur

Association Agôn

Référence électronique

Barbara Métais-Chastanier, «Pouvoir et Corps : quel corps? », Agôn [En ligne], Critiques, mis en ligne le 09 février 2019, consulté le 23 septembre 2020. URL : http://journals.openedition.org/agon/2275 ;

DOI : https://doi.org/10.4000/agon.2275

Ce document a été généré automatiquement le 23 septembre 2020.

Association Agôn et les auteurs des articles 


\title{
Pouvoir et Corps : quel corps?
}

\author{
Jusque là c'est nous, Matthieu Hocquemiller
}

\author{
Barbara Métais-Chastanier
}

\section{RÉFÉRENCE}

Jusque là c'est nous, Matthieu Hocquemiller

Les 5 et 6 juillet au festival Montpellier Danse 2012.

« Le pouvoir s'est avancé dans le corps, il se trouve exposé dans le corps même. » Michel

Foucault.

1 Un corps en cache toujours un autre, voilà ce qu'au sortir de Jusque là c'est nous de Matthieu Hocquemiller présenté les 5 et 6 juillet 2012 dans le cadre de la $32^{\mathrm{e}}$ édition du festival Montpellier Danse, il peut y avoir non pas comme constat, mais comme évidence, comme constante révélée dans et par des corps, et cela de manière toujours délicate, sensible et efficace, par le retrait d'une chose présentée qui en contiendrait d'autres.

Un corps en cache toujours un autre :

Cette image de la traversée de la scène, d'abord, - reprise et répétée par la traversée simultanée d'une autre danseuse - qu'on peine pourtant à voir, silhouette plus lointaine, doublant la première, vue et oubliée sitôt apparue, qui pourtant fait trace. On ne sait pas ce qu'on a vu. Toujours derrière le premier corps se trouve un autre corps qui semble faire retraite.

4 Cette image ensuite, qui la précède en réalité, mais c'est au moment de la traversée qu'on la réalise, comme après coup: dans les deux corps qu'on avait vaguement identifiés comme étant l'un masculin et l'autre féminin, la nudité - brusque - lacunaire - presque forcée et soustraite aussitôt consentie - découvre l'autre corps qui se tenait derrière le premier (qu'on arrête au visage, aux signes apparents et manifestes du corps visible et donc lisible), un corps où la partie n'est pas signe du tout, qui existerait comme ensemble indécomposable mais toutefois composé d'éléments empruntés à l'un et 
l'autre des deux sexes : ils sont deux, de part et d'autre, adossés aux panneaux blancs qui cadrent le regard et dessinent dans l'espace quelque chose comme une série d'avancées et de scènes possibles pour des apparitions. Ils sont deux, alternativement éclairés par une bande lumineuse qui parcourt de gauche à droite le visible comme un scanner qui viendrait fouiller et révéler - sans pour autant permettre de les voir - les aspérités qui composent l'obscurité. Ils sont deux et une lecture paresseuse, empêtrée d'habitudes et qui ne vient même pas s'énoncer comme identification (là encore, ce n'est qu'après qu'on le remarque), vous fait très rapidement placer la femme côté jardin et l'homme côté cour. Ils sont deux, font ce geste-là, ce geste simple d'ôter leurs vêtements, haut et bas, mais jamais complètement, non : ils s'arrêtent dans le geste, le retiennent, et quand le scanner revient, que la bande lumineuse passe à nouveau sur leurs corps, on découvre la nudité, les emboîtements possibles des sexes qui font corps et composent un autre possible. Derrière la femme, il y a toujours l'homme possible, derrière l'homme, la femme, et toutes les formes de continuité éventuelles de l'un à l'autre.

5 Dernière image enfin, cet homme ou cette femme dont le corps déborde tout ancrage sexuel, manipulé/e par les trois autres danseuses, invité/e à prendre des poses et des positions qui déjouent, tout autant qu'elles les reprennent, les attendus de l'exploitation économique et symbolique de l'érotisme féminin: corps de la femme sexualisé en tant qu'il est objet de désir et qu'il se donne en image, mais livré là, avec une grande douceur, de manière à la fois violente et retenue, sous les abords d'un trouble qui s'infiltre autant dans le genre que dans sa perception, abandonné aux gestes précis et minutieux de celles qui le font se plier à l'exercice de la composition d'un corps social - jambes croisées en position assise, déhanché accentué en position debout, cambrure parfaite quand il faut s'allonger, violence faite au corps par le visible et par le regard qui se révèle au moment où l'interprète, devenu pantin rouge et sans volonté, livre au spectateur les lignes du sexe absent à-demi visible sous la matière tendue des plis du justaucorps. Mon corps, c'est d'abord ce à travers quoi passe le langage de tous les corps, la circonscription des identités et des comportements dans la chair de ce que je crois m'être le plus propre: le corps de l'autre, le corps de tous les autres (social) s'incarne et s'exprime toujours dans mon corps propre.

Alors bien sûr pour commencer il aurait d'abord fallu dire que Matthieu Hocquemiller est un chorégraphe montpelliérain, qu'il a fondé en 2004 la compagnie chorégraphique contemporaine «A contre poil du sens » (A.C.P.S.), qu'il présente ici sa sixième création (après entre autres Post disaster dance, Bonnes Nouvelles et J'arrive plus à mourir), qu'il a un pied dans l'université (formation en sciences humaines), un autre en acrobatie et en arts du cirque, et un autre enfin (ce qui fait donc au total trois pieds) dans le milieu libertaire et le mouvement altermondialiste, qu'il n'a de cesse de revenir sur la manière dont le corps révèle toujours d'une politique du corps, qu'il travaille ici avec quatre danseurs (Jeanne Nora Bennouar, Mathilde Gautry, Hurlande Véronique Seukeu, Ludovic Lézin), dans une démarche qui emprunte à la fois au journalisme - il y a derrière cette pièce tout un travail documentaire d'investigation nourri et altéré par des rencontres - et à la promenade - la scène avance comme se recompose un paysage, au gré des points de vue - avec une question liminaire qui pourrait être celle-ci: «Comment des corps habitent une époque et sont habités par elle?»

7 En effet, il aurait fallu écrire tout cela pour ensuite revenir à la pièce elle-même, courte et saisie, qui s'ouvre sur ces silhouettes découpées par la lumière - où un corps apparaît 
en creux, à travers ce qu'on en découvre - quatre donc, et ce qui fait apparaître l'interprète ce n'est pas ici la masse, le poids, les mouvements respectifs, mais bien l'enveloppe extérieure : je suis parce que je suis surface, voilà ce que nous disent ces quatre formes suspendues. Surface sensible, surface intelligible, surface de moi et d'autres moi. Surface possible comme visage et comme projection d'un autre visage possible. Surfaces. Ce n'est qu'après que survient la lumière, bande étroite qui circule de gauche à droite, rayon dans lequel se découvre le danseur. Ici le corps n'entre pas. Il apparaît et s'efface. Tout s'ordonne autour des conditions de son apparition : le travail de Hocquemiller emprunte à l'ordre du surgissement. Et toujours l'œil cherche à voir ce qu'il croit avoir vu. Un sexe, une nudité. Et alors doute. Revient. Cherche à se rappeler mais le voici déjà rattrapé par le retour des mêmes images, qui pourtant toujours diffèrent. Le jeu tient à une manipulation froide et maîtrisée de l'érotisme de la fragmentation du corps (une partie) ou du regard (un instant) : le « on ne sait pas ce qu'on voit » devenant peu à peu, au fil de la pièce et tandis que les corps s'éclairent, un " on ne sait pas qui on regarde ». Moitiés de corps, moitiés de geste, moitiés de vision, bribes et éclats. Instants. Le corps est isolé. Ce n'est qu'après qu'il rencontre l'autre. Le fragment devient corps appuyé, corps reposé, corps toujours objet donc (soit par le traitement optique - fragment -, soit par le traitement physique - manipulation) ou plutôt offert, dans sa fragilité, au souci et à l'attention de l'autre, corps moins objectivé qu'adossé, à la fois choyé et rejeté. A la manière des enfants ou des vieillards qui bouleversent et irritent parce qu'ils ont besoin d'autrui, ici le corps conduit est tout à la fois objet de soin et d'agacement : la vulnérabilité exige tout autant qu'elle indispose.

Jusque là c'est nous, Matthieu Hocquemiller

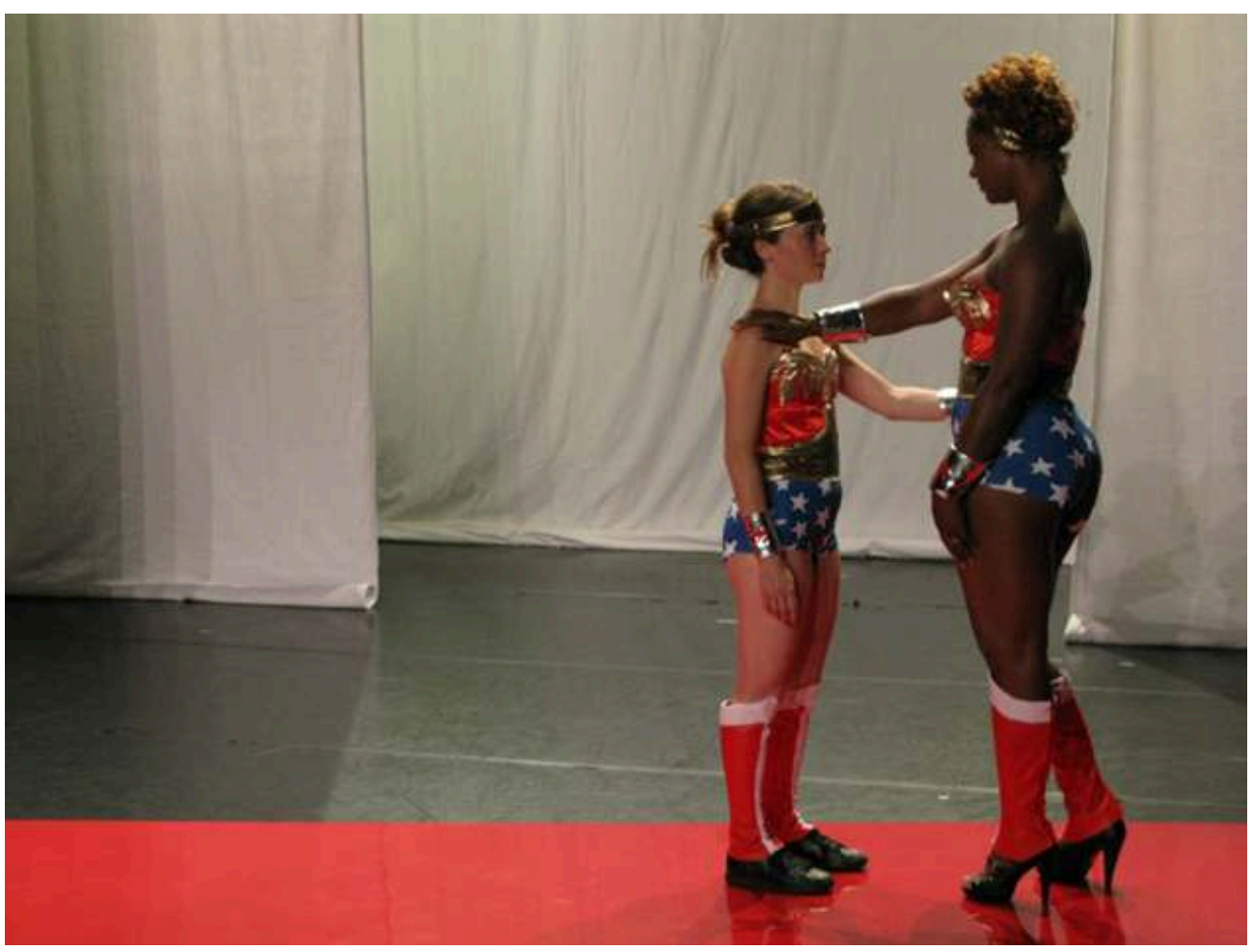

(c) Compagnie A contre poil du sens

8 A la délicatesse de la manipulation, succède donc le costume de la maitrise dans son versant superlatif: celui du super-héros, ou plutôt de la super-héroïne l'héroïne 
générique, indépassable, in-surpassée, Wonder Woman bien évidemment : short bleu avec étoiles et bustier rouge flashy donnant le ton de ce passage des enjeux identitaire et singuliers à d'autres plus spécifiquement sociaux et collectifs. Mais de même que les pauses restaient en surface sur le premier corps manipulé, là aussi le costume reste ce qu'il est, à savoir une surface dont on ne sait pas quoi faire ${ }^{1}$ : les deux interprètes (Mathilde Gautry et Hurlande Véronique Seukeu), chacune équipée de son kit paillettes et bustier push-up, sagement placée sur la diagonale du tapis rouge qui cisaille la scène tout autant qu'il la tire ailleurs que du côté de la frontalité, se livre à des portés très lents, très doux et très légers, ou c'est tantôt l'une qui devient appui de l'autre, puis impulse une marche, déclenche un déplacement - réversibilité discrète des gestes de l'une et de l'autre, aux corps si évidemment dissemblables, qui révèle ironiquement l'absence du corps grandiose, doublement parfait : dans sa plastique sculpturale comme dans son comportement - Wonder Woman, "All the world is waiting for you / And the power you possess. / In your satin tights, / Fighting for your rights, / And the old red, white, and blue $e^{2}$ »

Dans un entretien sur les rapports qui unissent le corps au pouvoir, Michel Foucault, à la question "Quelle est l'évolution du rapport corporel entre les masses et l'appareil d'État?", concluait sa réponse par le constat suivant: "Reste à étudier de quel corps la société actuelle a besoin ${ }^{3} . .$. ». Matthieu Hocquemiller se livre à l'étude moins de celui dont elle a besoin que de celui qu'elle ne veut pas voir et encore moins donner à voir. Avènement d'un corps qui ne serait pas donné ? Il reste encore à venir.

\section{NOTES}

1. Dans le prolongement de cette idée d'un corps surface, voir le court-métrage (j!) réalisé par Matthieu Hocquemiller en juin 2009. URL : http://vimeo.com/24181659

2. Premier couplet du générique de la série Wonder Woman.

3. Michel Foucault, "Pouvoir et corps " (in Quel corps?, n², septembre-octobre 1975, pp. 2-5., entretien de juin 1975), texte n¹57, in Dits et Ecrits, vol. II (1970-1975), Gallimard, 1994, p. 756.

\section{INDEX}

Mots-clés : Hocquemiller (Matthieu), Métais-Chastanier (Barbara), Montpellier, Danse, corps, Jusque là c'est nous 